\title{
Perfil profesional del egresado de ingeniería de sistemas e informática por competencias
}

Professional profile of the graduate engineer of systems and computer by computers

Marisol Josefina Sarmiento Alvarado msarmiento@usdg.edu.pe https://orcid.org/0000-0003-3792-5878 Universidad Santo Domingo de Guzmán

José Gregorio Brito Garcías jbrito@usdg.edu.pe

https://orcid.org/0000-0001-8999-8126

Universidad Santo Domingo de Guzmán

\section{Resumen}

Esta investigación se desarrolló en el marco del diseño de perfiles profesionales por competencias en el área de estudios de la Informática, cuyo objetivo fue construir el perfil profesional del egresado de la Carrera Profesional de Ingeniería de Sistemas e Informática de la Universidad Santo Domingo de Guzmán. Esta investigación surgió de la necesidad de revisar el diseño actual del perfil profesional del egresado en este programa de estudio y compararlo con las exigencias laborales competitivas de esta nueva sociedad peruana, a través del análisis ocupacional y teniendo como principio la visión del profesional afín a esta carrera. El estudio se ubicó dentro de una visión postpositivista de la realidad, enfocándose en la Teoría Fundamentada, con el método comparativo continuo y apoyo de la hermenéutica. En relación con los sujetos informantes se trabajó con los estudiantes del último ciclo, docentes especialistas y diversas empresas relacionadas con Sistemas e Informática, a fin de conocer la demanda laboral actual. Como instrumento para la recolección de la información se utilizó la entrevista semiestructurada, se construyeron los textos protocolares de donde emergió la categoría sol de la investigación y sus subcategorías, ello permitió diseñar la matriz de análisis ocupacional que decantó en el perfil profesional del egresado. A partir de los resultados se presenta una propuesta apegada a las demandas del mundo moderno, transdisciplinario y fundamentado en la formación integral del estudiante y cuyo proceso educativo está centrado en el estudiante, en una sociedad permeada por la tecnología.

Palabras clave: Perfil profesional del egresado, competencias, análisis ocupacional, ingeniería de sistema e informática, USDG. 


\begin{abstract}
Summary
This research was carried out within the framework of the design of professional profiles by competencies in one of the areas of significant studies, such as Computer Science, whose objective was to build the professional profile of the graduate of the Professional Career of Systems and Computer Engineering of the Santo Domingo de Guzmán University. This research arises from the need to review the current design of the professional profile of the graduate in this study program and compare it with the professional labor demands of this new Peruvian society, through occupational analysis and based on the vision of the professional related to this career. The study was located within the post-positivist paradigm and its qualitative approach, from hermeneutics and using the grounded theory, the method used was the continuous comparative, as informants we worked with the students of the last cycle, specialized teachers and various companies related to Systems and Computer science, in order to know the current labor demand, as an instrument for the collection of information, the semi-structured interview was used, the protocol texts were created from which the sun category of research emerges and its subcategories, this allowed to design the matrix of occupational analysis that decays the professional profile of the graduate. Based on the results, a proposal is presented in accordance with the demands of this modern, trans-disciplinary world based on the integral formation of the student and whose educational process is centered on the student, in a society permeated by technology.
\end{abstract}

Keywords: Professional profile of the graduate, skills, occupational analysis, system engineering and computer science, USDG. 


\section{Introducción}

En la educación universitaria del siglo XXI la megatendencia, entre otros elementos, es la formación integral por competencias, la inclusión y uso de las tecnologías, como procesos de alfabetización e innovación, tal como está contemplado en la Organización de las Naciones Unidas para la Educación, la Ciencia y la Cultura (UNESCO, 1998) al señalar que:

La Educación para Todos y la Educación para Toda la Vida se presentan hoy en día como dos grandes retos educativos que al mismo tiempo que orientan a los educadores en su labor docente, plantean nuevos horizontes profesionales, y en consecuencia, generan la necesidad de una formación adecuada y acorde con la importancia de su misión educativa. Por otra parte, los cambios que se avecinan como consecuencia de las nuevas tecnologías de la información, la mundialización de la economía, y los avances científicos y tecnológicos que protagonizan esta última década, configuran hoy en día un nuevo contexto que hacen de la educación una cuestión de vital importancia tanto a nivel individual como a nivel social. (p. 267).

Por lo cual se hace necesario la reconstrucción de las principales tendencias de la educación, las políticas, las metodologías de enseñanza y los enfoques pedagógicos que puedan influir para transformar la realidad educativa en cada contexto tanto local como mundial que propenden el desarrollo económico, político, social y cultural que ha de darse en los años venideros.

De esta manera, es importante tener presente el tratado de Bolonia (1999) que nace con la intención de reestructurar los sistemas de la superior en Europa y la convergencia del mismo, para la mejora del empleo, la movilidad de ciudadanos, la competitividad internacional y la declaración de competencias. Para ello se establece el Proyecto Tuning en el año 2000, el cual se centra en el estudiante y en el desarrollo de las competencias tanto personales como profesionales. En el año 2004 es cuando se crea para Latinoamérica el desarrollo integral del currículo basado en competencias a través del Proyecto Alfa Tuning; al identificar e intercambiar información para la mejora y colaboración entre las instituciones de Educación Superior y el desarrollo de la calidad, efectividad y transparencia de la educación.

Es así como la UNESCO y la Organización de las Naciones Unidas (ONU) están de acuerdo con que la educación debe reorganizarse en sus estructuras de enseñanza, en el aprendizaje y prácticas en el aula a partir de las denominadas Competencias del siglo XXI, no como una necesidad del futuro sino como en el presente para poder asegurar el desarrollo y éxito profesional de las personas y por ende el de las naciones. Según la UNESCO (2007, p.2) “la elección de la Contacto: revistaeduca@umch.edu.pe ISSN : 2617-0337 revistas.umch.edu.pe/ 
competencia como principio organizador del curriculum es una forma de trasladar la vida real al aula". Es decir el proceso educativo desarrollado a través de competencias asume la realidad de la sociedad del conocimiento y la era digital.

En palabras de (Braslavsky, 1999, p.22) la competencia es el desarrollo de las capacidades complejas por medio de las cuales los estudiantes pueden pensar y actuar en diversos espacios. “...consiste en la adquisición de conocimiento a través de la acción, resultado de una cultura de base sólida que puede ponerse en práctica y utilizarse para explicar qué es lo que está sucediendo...". Es hacer algo en un contexto de manera idónea. Para la ONU (2016) es la forma más idónea de preparar al estudiante en su presente y para el futuro. Por lo cual es esencial concentrar los esfuerzos en la Educación con Competencias.

Ello implica que, en un currículo orientado por competencias, el perfil de un educando determina los tipos de circunstancias que los estudiantes tienen que ser capaces de solucionar de forma eficaz, al final de su formación. Es decir, en situaciones referentes a la vida real, al mundo laboral o a su propia disciplina. Esto implica que toda institución educativa está obligada con el desarrollo individual, tomando en consideración la diversidad y la equidad, el convivir juntos y la adaptación a los cambios de las nuevas sociedades.

Sin duda alguna le atañe a la educación universitaria replantear sus diseños curriculares e imbricar los nuevos escenarios: la globalización, la revolución tecnológica, la multiculturalidad e identidad nacional, los enfoques educativos centrados en el estudiante, formación docente acorde con el conocer-saber y la atención a lo transdisciplinar. (Tejada, 2005). En este sentido Zabalza (2003) afirma que los docentes universitarios deben permanecer actualizados en su formación académica y profesional, en el manejo de lo instrumental, en el acceso a la información de punta, la investigación, la extensión, lo curricular y lo didáctico.

En consideración se debe definir qué es un currículo por competencias; no sin antes conceptualizar qué es un currículo, citando a (Stenhouse, 1984) lo concibe no solo como un sucinto agrupamiento de asignaturas que se abordan desde la práctica pedagógica, en conjunto con los diversos objetivos, métodos y contenidos, sino que además es una antropología, una filosofía de la educación, un paradigma pedagógico. Es decir, es todo aquello que orienta y da sentido a la organización y a la acción pedagógica concreta. La educación es una ciencia y un arte y el contenido sustantivo del arte de la enseñanza y del aprendizaje es el curriculum (Rudduck, 1998).

Contacto: revistaeduca@umch.edu.pe ISSN : 2617-0337 revistas.umch.edu.pe/ 
Existen diversos enfoques curriculares, el currículo bajo la concepción académicatradicional, la concepción conductista, la perspectiva socio-critica-liberadora, el currículo entendido como conjunto de experiencias, el currículo centrado en el estudiante y el currículo por capacidades y competencias.

Con respecto al currículo por capacidades y competencias, centra su importancia en el capital-talento-humano y cuya formación educativa debe responder a las necesidades que demanda este mundo moderno y globalizado. Es decir ha de “...potenciar las capacidades naturales de los estudiantes, el pensar, el sentir, el actuar y el transcender...”. Como formación y desarrollo de “...competencias integrales-sistémicas, interpersonales e instrumentales que se concretan a través del saber-conocer, el saber relacionarse con los demás, el saber hacer y el saber trascender (ser)...”. (Delors, citado en Latorre, 2016, p. 36).

El diseño curricular a partir de competencias, posee una metodología que difiere según los diversos autores, en este trabajo de investigación se asume la metodología planteada por Santiváñez (2013) quien precisa las siguientes etapas y de manera específica el literal b:
a) Marco teórico y doctrinario.
b) Perfil profesional del egresado.
c) Organización del currículo.
d) Estrategias didácticas.
e) Evaluación y Acreditación del grado y título profesional.
f) Estrategia de aplicación del Plan Curricular.

La educación del siglo XXI, se caracteriza por el desarrollo de un currículo integral basado en competencias, por el estudio centrado en el estudiante, el reconocimiento de la investigación y la responsabilidad social universitaria, la diversidad cultural y lingüística, docentes competentes, así como también por la sociedad del conocimiento. Por lo cual toda institución educativa de nivel superior debe permanecer actualizada y establecer reingenierías, procesos de mejora continua y de evaluación de todo su sistema educativo, a fin de responder a las demandas de esta nueva sociedad permeada por lo complejo, lo transdisciplinar y por lo transversalizado en el uso de las Tecnologías de la Información y la Comunicación (TIC).

Con respeto a las TIC, la Informática es una de las carreras con amplia demanda, tanto como área especializada o como área de apoyo al resto de las áreas, en el ejercicio profesional y en el desarrollo de conocimiento científico. Por lo cual, es preciso que las universidades dedicadas a la Contacto: revistaeduca@umch.edu.pe ISSN : 2617-0337 revistas.umch.edu.pe/ 
preparación de estos profesionales, evalúen sus perfiles de formación y los adapten a las crecientes y nuevas demandas del mercado empleador. En el caso de la Universidad Santo Domingo de Guzmán, se tiene una Decanatura de Ingeniería, la cual está conformada por dos carreras profesionales; Ingeniería de Negocios e Ingeniería de Sistemas e Informática la cual tiene como Perfil de egreso el siguiente:

- Posee una vocación definida por su Programa de Estudios con probada inteligencia emocional.

- Asume responsablemente el riesgo de sus opiniones, basadas en la información recibida y contrastándolas con sus conocimientos adquiridos.

- Posee liderazgo personal y liderazgo organizacional.

- Habilidad para desempeñarse en el campo laboral, aplicando sus conocimientos con coherencia y basados en fundamentos lógicos.

- Es un profesional prudente, justo y ponderado en sus opiniones.

- Es disciplinado, puntual y responsable.

- Perseverante, creativo, pionero de ideas, culto, posee una ética, valores y principios.

- Respeta el pensamiento divergente y valora la interculturalidad, asumiendo una actitud tolerante.

- Hábil para conducir equipos de trabajo con pensamiento y paradigmas divergentes.

- Buen dominio teórico de los fundamentos y principios que sustentan a la Ingeniería de Sistemas.

- Posee una visión de la realidad de su país y del mundo.

- Realiza procesos de planeación, administración y evaluación de programas y proyectos innovadores.

- Propone alternativas de solución a problemas de la sociedad actual.

- Tiene disposición para ampliar su propio horizonte.

- Con capacidad para seleccionar y utilizar nuevas tecnologías de manera contextualizada.

- Desarrolla proyectos de investigación científica en ingeniería.

Con base en lo antes señalado es necesario establecer si este perfil del egresado está cumpliendo con la formación de profesionales altamente calificados y de cara a las exigencias de 
las megatendencias en educación, en la formación integral, que permita insertar en la sociedad profesionales competentes en el área.

De esta manera, surgieron las preguntas de investigación a partir de las cuales, se plantearon los objetivos de investigación; el objetivo general fue construir el perfil profesional del egresado de la Carrera Profesional de Ingeniería de Sistemas e Informática por competencias de la Universidad Santo Domingo de Guzmán, teniendo como principio la demanda profesional de la Sociedad Peruana. Los objetivos específicos fueron: caracterizar la demanda profesional del Ingeniero de Sistemas e Informática en la Sociedad Peruana; construir el análisis funcional de la Carrera Profesional de Ingeniería de Sistemas e Informática de la universidad y comparar la funcionalidad de la Carrera Profesional de Ingeniería de Sistemas e Informática de la universidad con la demanda profesional de la Sociedad Peruana.

De esta manera, un diseño curricular se elabora con la finalidad de orientar la práctica educativa, como un proceso dinámico, continuo y participativo, en el cual se analizan las características, condiciones y necesidades del contexto social, económico y político y por ende educativo, a fin de establecer los fines y objetivos educativos según el estudio realizado, se debe poner en práctica los contenidos, procedimientos y métodos de aprendizaje en conjunto con los recursos humanos, informáticos, financieros y organizacionales, $\mathrm{y}$ de forma continua se ha de evaluar la eficacia del mismo.

Por lo cual, es importante el desarrollo de toda investigación que permita evaluar a los profesionales egresados de una universidad en concordancia con las necesidades de la sociedad actual y a través de herramientas novedosas y efectivas. De esta manera, esta investigación es un aporte ya que realizó el estudio de una realidad social y educativa, presentó un diagnóstico y a partir del mismo elaboró el diseño de un perfil del egresado de la universidad estudiada.

Desde el ámbito económico y social, responde a una necesidad de formación de profesionales altamente competentes pertenecientes a una sociedad a la cual deben insertarse de manera idónea y contribuir social y económicamente en el progreso de su país. A nivel investigativo, ha de servir de base para nuevas investigaciones y en la consolidación de métodos científicos demostrativos de la realidad educativa que se gesta en la sociedad moderna.

Es importante mencionar algunos estudios previos nacionales e internacionales que dieron sustento a esta investigación, tales como la investigación de Pastor (2016) sobre el Perfil del Ingeniero Industrial Actual en el Perú a Partir del Modelo Tuning Latinoamérica y Molina (2000) Contacto: revistaeduca@umch.edu.pe ISSN : 2617-0337 revistas.umch.edu.pe/ 
titulada "La competencia profesional en el ingeniero del nuevo milenio", que tuvo como objetivos analizar, definir, sintetizar y consolidar un conjunto de competencias profesionales realizado para Latinoamérica al perfil del egresado de una carrera profesional. Y plantean cuatro grandes esferas en la formación profesional: Aprender a conocer, aprender a hacer, aprender a convivir y aprender a ser.

Para efectos del trabajo de investigación realizado, se asumió la postura de Guardián (1979), Santiváñez (2013) y Casarini (2017) los cuales plantean la importancia de conocer y desarrollar cada elemento para poder establecer un diseño curricular, entre ellos se tienen: a) Los fundamentos curriculares (Marco Teórico Doctrinario); que son las bases teóricas en que se sustentan los conceptos y concepciones curriculares. b) El perfil educativo (Perfil de Ingreso y Egreso); que es el conjunto de rasgos de personalidad, de conocimientos, de habilidades y destrezas, que se espera sean alcanzados por los participantes de un programa educativo dado. c) La organización del currículo (Áreas Curriculares); que comprende el plan de estudio. d) Las estrategias didácticas (Estrategias de Enseñanza y de aprendizaje). e) El sistema evaluación y acreditación (Evaluación de los Procesos de Enseñanza y de Aprendizaje); la normativa para evaluar el currículo establecido. f) Las estrategias de aplicación (Equivalencia y Normas); normas de aplicación del mismo.

Y se asumió en referencia a la metodología para diseñar un plan curricular, solo el literal b) El perfil educativo (Perfil de Ingreso y Egreso), que constituye el objetivo de esta investigación; y se conceptualiza como:

El conjunto de las competencias genéricas y específicas que la institución educativa va a formar en los estudiantes, a partir de la revisión de la profesión y del estudio de actividades y problemas hallados en el análisis del contexto disciplinar, investigativo, social y profesional-laboral. Dicho perfil orienta todos los planes de estudios, las estrategias didácticas y los procesos de evaluación, pues en él se describen, con la suficiente precisión, las competencias que se esperan formar al término de los estudios de una carrera determinada (García, como se citó en Santivañez, 2013, p. 70).

La metodología utilizada por (Santivañez, 2013, p. 70), presenta cinco (05) pasos para diseñar el Perfil del egresado tomando como base lo planteado por Valdivia (1994), García y Tobón (2010), y es la metodología que se tomó en el desarrollo de esta investigación:

1) Diagnóstico de la demanda profesional.

1.1 Caracterización de la sociedad y de sus perspectivas de desarrollo.

1.2 Oferta actual de recursos calificados.

1.3 Áreas prioritarias en la demanda de los recursos humanos calificados. Contacto: revistaeduca@umch.edu.pe ISSN : 2617-0337 revistas.umch.edu.pe/ 
1.4 Demanda de recursos humanos.

2) Determinación de los componentes del perfil profesional del egresado.

2.1 Análisis y diagnóstico de la nueva actividad de la profesión a nivel macro y micro.

2.2 Análisis y diagnóstico de las instituciones.

3) Determinación de la metodología de análisis ocupacional.

4) Formulación del perfil genérico del profesional egresado.

5) Formulación del perfil específico del egresado a partir de competencias.

En palabras de (Santivañez, 2013, p. 62) define al perfil del egresado como el conjunto de capacidades y atributos que la institución formadora se compromete a desarrollar en sus estudiantes al finalizar un Plan de Estudios y lograr un nivel apropiado que les permita insertarse exitosamente en el ámbito laboral o en el sector productivo.

Es decir son la agrupación de las diferentes competencias que delinean el Plan de Estudios, la evaluación y los objetivos finales de una carrera. En cuanto a los perfiles, el genérico; agrupa las competencias y capacidades concernientes a la actividad humana, las mismas pueden ser comunes para varias profesiones. El perfil específico; comprende aquellas competencias conexas con los aspectos técnicos de cada profesión que no se pueden ceder a otras profesiones u otros contextos laborales; estas comprenden habilidades y destrezas que han de desempeñarse o desarrollarse a través de actividades que involucra o exige una mayor formación teórico-práctico.

Para la construcción o diseño del perfil del egresado a partir de competencias, se debe desarrollar la matriz de análisis ocupacional de la profesión, el perfil genérico y el perfil específico (matriz de competencias y desempeños específicos). Con base en estos planteamientos se detalla la metodología:

a) Diagnóstico de la demanda profesional

a.1.- Caracterización de la sociedad peruana y de sus perspectivas de desarrollo; considerando la situación actual de la sociedad, la proyección económica del país y la estrategia nacional de desarrollo.

a.2.- Oferta actual de recursos calificados; considerando las matrículas universitarias, la población activa y los sectores prioritarios para la oferta.

a.3.- Áreas prioritarias en la demanda de recursos humanos.

Contacto: revistaeduca@umch.edu.pe ISSN : 2617-0337 revistas.umch.edu.pe/ 
a.4.- Demanda de recursos humanos; demanda de los recursos humanos y prioridad de formación profesional.

b) Determinación de los componentes del Perfil Profesional del Egresado; se estructura con base en dos componentes:

b.1.- Análisis y diagnóstico de la nueva actividad de la profesión, a nivel macro y micro (profesionales exitosos, empleados y clientes).

b.2.- Análisis y diagnóstico de la institución; organización y el contenido curricular, personal docente especializado, infraestructura y tecnología disponible para la formación profesional.

c) Determinación de la Metodología de Análisis Ocupacional; a través del desarrollo de los puestos de trabajo y las tareas. A fin de diseñar la matriz de análisis ocupacional.

d) Formulación del perfil genérico; conformado por las competencias genéricas.

e) Formulación del perfil específico; son las capacidades conceptuales, habilidades y actitudes propias de cada profesión, tomando en consideración lo humano, lo técnico y la investigación.

e.1.- Elaboración de las matrices de competencias y desempeños específicos; se elabora según competencias y desempeños para cada área curricular, según lo levantado en la matriz de análisis ocupacional.

e.2.- Formulación de las competencias específicas del perfil; luego de analizar la matriz ocupacional se formulan las competencias específicas.

e.3.- Formulación de los desempeños específicos de cada competencia específica.

\section{Métodos}

Esta investigación, por sus características y propósito, se enmarcó en una visión postpositivista, en virtud de garantizar el acercamiento a la certeza de la comprensión, descripción y caracterización del objeto de estudio -Perfil por Competencias para la Carrera Profesional de Ingeniería de Sistemas e Informática- tomando en consideración ontológicamente al ser humano. El termino comprensión, en esta perspectiva, es utilizado por diferentes autores, donde se destaca Maturana (1997) quien establece la comprensión del fenómeno a estudiar.

La ubicación en la perspectiva postpositivista, a la que diversos autores incluyen en una visión cualitativa, se apoyó en lo planteado por Taylor y Bogdan (1986); Maturana (ob. Cit); Martínez Contacto: revistaeduca@umch.edu.pe ISSN : 2617-0337 revistas.umch.edu.pe/ 
(1992) quienes plantean que en esta visión se extraen descripciones a partir de las observaciones que adoptan la forma de entrevista, narraciones, notas de campo y transcripciones (LeCompte, 1995). De igual manera la investigación cualitativa produce datos descriptivos a partir de las propias palabras de las personas y las conductas observables (Taylor y Bogdan, 1990).

Así mismo para efectos de la investigación, en cuanto a la Teoría Fundamentada así como el método de comparación constante se complementan con la hermenéutica por la naturaleza epistémica que ofrece la visión postpositivista con el fin de develar, describir, interpretar, el sentido de los mensajes (verbalizaciones) y las interconexiones textuales. Es así como Ríos (2005) expone lo hermenéutico, como un camino de investigación educacional que puede constituirse en un nuevo estilo de investigación cualitativa el cual ofrece una convergencia que complementa los aportes de otras investigaciones que tratan las problemáticas emergentes en el sistema educativo.

Desde el enfoque comprensivo interpretativo que ofrece la hermenéutica, se abre un camino develador de sentidos que permanecen encubiertos en las acciones y los discursos de los sujetos investigados. El estilo de la investigación permite combinar el enfoque de la Teoría Fundamentada en Datos de Glasser y Strauss, (1999) y Pandit (1995), ya que en el proceso descriptivointerpretativo se combinan los conceptos, las categorías, subcategorías, proposiciones que deriven de los escenarios educativos estudiados (Universidad formadora de Ingenieros en Sistemas e Informática y Espacios laborales). En la Teoría Fundamentada los conceptos constituyen la unidad básica de análisis y según Corbin y Strauss $(2002$, p. 7) “...es desde la conceptualización de los datos y no desde los datos en sí, donde se desarrolla la teoría...”.

El método comparativo continuo o constante es inherente a la investigación cualitativa creado por Glasser y Strauss (Ob. cit) su uso radica en que permite sistematizar la información para generar teoría de manera cualitativa, entrelazando tres momentos: recogida de información, análisis de información e interpretación de la información, lo cual permite trabajar de manera dialéctica a través de comparaciones sucesivas entre la realidad, el contexto, la información y la teoría para obtener una formulación teórica sólida, pertinente, integrada y contextualizada de la realidad investigada.

El contexto de estudio de esta investigación estuvo representado por la Universidad Santo Domingo de Guzmán, en la carrera profesional de Ingeniería de Sistemas e Informática. Teniendo como informantes a cinco (05) docentes que administran asignaturas del componente específico y de la especialidad, a veinticinco (25) estudiantes de los últimos tres ciclos. En cuanto a la Sociedad Contacto: revistaeduca@umch.edu.pe ISSN : 2617-0337 revistas.umch.edu.pe/ 
Peruana, se tuvo como informantes a siete (07) profesionales empresarios y gerentes relacionados con la Ingeniería de Sistemas e Informática.

Se utilizó como técnica de recolección de información, la entrevista semiestructurada con base en una serie de preguntas directrices contentiva de los "cómo y los qué es" como aspectos que ordenan la entrevista. "Esta apreciación se deriva del enfoque construccionista y expresa que el proceso de construcción del significado es tan importante como el significado que se construye" (Rodríguez y García, Ob cit), así como el método del análisis ocupacional sustentado por Santiváñez (2013).

\section{Resultados}

El contexto de estudio representado por la Universidad Santo Domingo de Guzmán, en la carrera profesional de Ingeniería de Sistemas e Informática, tuvo como informantes a los docentes que administran asignaturas del componente específico y de la especialidad, a los estudiantes de los últimos tres ciclos. En cuanto a la Sociedad Peruana, se tuvo como informantes a los profesionales empresarios y gerentes relacionados con la Ingeniería de Sistemas e Informática.

Para la obtención de la información se utilizó la técnica de la entrevista semiestructurada, se contó con cinco (05) docentes, veinticinco (25) estudiantes y siete (07) profesionales empresarios y gerentes relacionados con la Ingeniería de Sistemas e Informática. Se procedió a la revisión de las entrevistas y a la construcción documental del análisis del texto escrito, se saturó con cinco (05) docentes, doce (12) estudiantes y cuatro (04) profesionales empresarios y gerentes relacionados con la Ingeniería de Sistemas e Informática. Al finalizar la revisión del texto de las entrevistas se obtuvo la categoría Sol denominada Perfil Profesional del Egresado de Ingeniería de Sistemas e Informática a partir de Competencias.

Con respecto al primer objetivo específico: Caracterizar la demanda profesional del Ingeniero de Sistemas e Informática en la Sociedad Peruana, a través de las entrevistas aplicadas se analizaron los protocolos de los textos a partir de las respuestas de los entrevistados y se concluyó de la siguiente manera:

a) Con respecto a las funciones del Ingeniero de Sistemas e Informática, a partir de la visión de los empleadores, en comparación con la visión de los profesionales y docentes y lo establecido en la universidad, se tiene:

Contacto: revistaeduca@umch.edu.pe ISSN : 2617-0337 revistas.umch.edu.pe/ 


\section{Tabla 1}

Funciones del Ingeniero de Sistemas e Informática

\begin{tabular}{|c|c|c|}
\hline Empleadores & Profesionales y Docentes & USDG \\
\hline 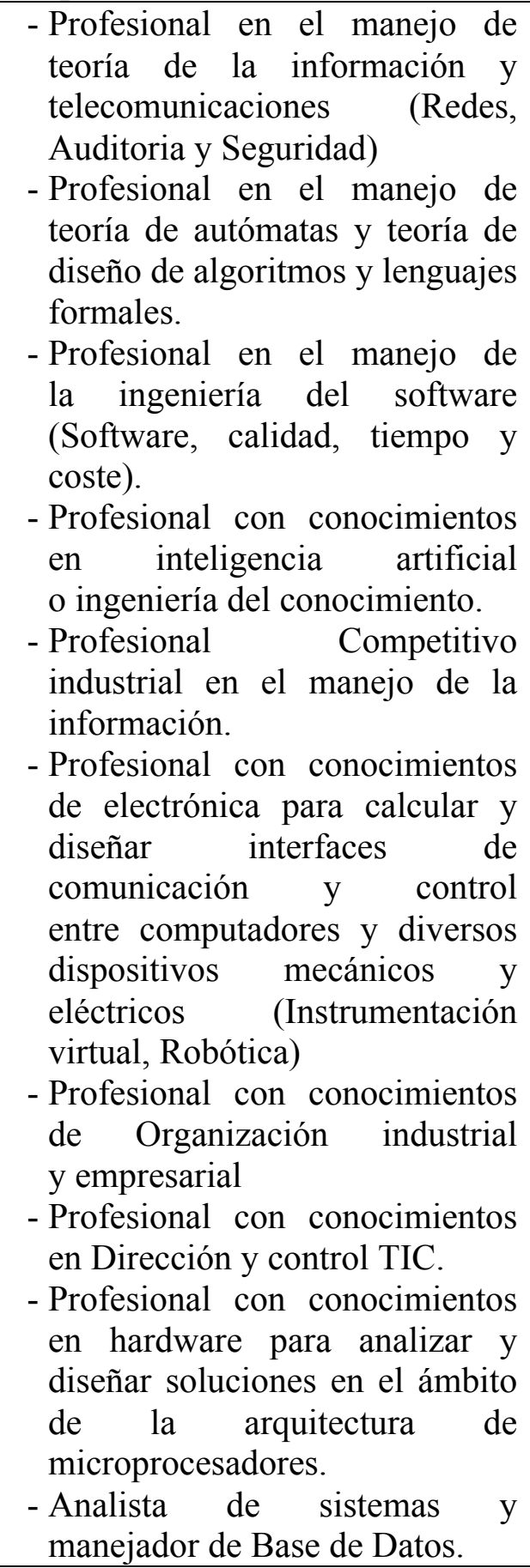 & $\begin{array}{l}\text { - Analizar las necesidades } \\
\text { requeridas } \\
\text { - Diseñar, programar, aplicar y } \\
\text { mantener (sistemas } \\
\text { informáticos, páginas web, } \\
\text { entre otros). } \\
\text { - Creador de aplicaciones } \\
\text { móviles. } \\
\text { - Administrar redes y sistemas } \\
\text { de información. } \\
\text { - Creador de videojuegos y } \\
\text { aplicaciones móviles. } \\
\text { - Encargado de plataformas de } \\
\text { e-commerce. } \\
\text { - Experto } \\
\text { Telecomunicaciones. } \\
\text { - Gestor de proyectos y } \\
\text { tecnologías. } \\
\text { - Analizar el rendimiento } \\
\text { económico, la efectividad de } \\
\text { los recursos humanos y el uso } \\
\text { tecnológico vinculado a sus } \\
\text { creaciones. } \\
\text { - Investigar para crear software } \\
\text { y hardware en una empresa u } \\
\text { organización. }\end{array}$ & $\begin{array}{l}\text { El Ingeniero de Sistemas e } \\
\text { Informática, egresado de la } \\
\text { Universidad Santo } \\
\text { Domingo de Guzmán, es el } \\
\text { profesional con sólida } \\
\text { formación humanística, } \\
\text { científica y tecnológica, } \\
\text { capacitado para: analizar, } \\
\text {-Planificar, ar an diseñar los } \\
\text { implementar y de información; } \\
\text { sistemas de in } \\
\text { - Asesor de áreas } \\
\text { informáticas; - Planear, } \\
\text { organizar y controlar los } \\
\text { sistemas de producción } \\
\text { tanto en la área de la } \\
\text { programación como en } \\
\text { las operaciones } \\
\text { vinculados a las } \\
\text { decisiones gerenciales; } \\
\text {-Organizar y entrelazar } \\
\text { redes electrónicas y vía } \\
\text { satélite, investigar en el } \\
\text { área de los ordenadores } \\
\text { informáticos. }\end{array}$ \\
\hline
\end{tabular}


b) Con respecto al campo laboral del Ingeniero de Sistemas e Informática, a partir de la visión de los empleadores en comparación con la visión de los profesionales y docentes y lo establecido en la universidad, se tiene:

\section{Tabla 2}

Campo laboral del Ingeniero de Sistemas e Informática

\begin{tabular}{|c|c|c|}
\hline Empleadores & Profesionales & USDG \\
\hline 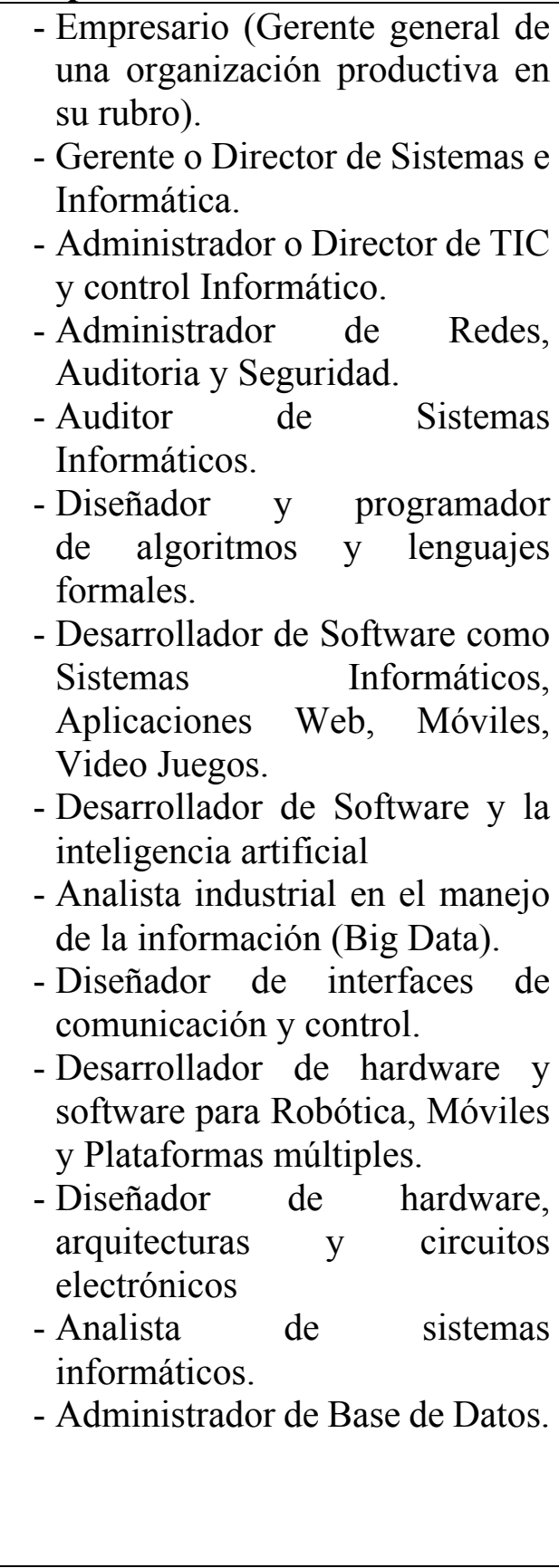 & 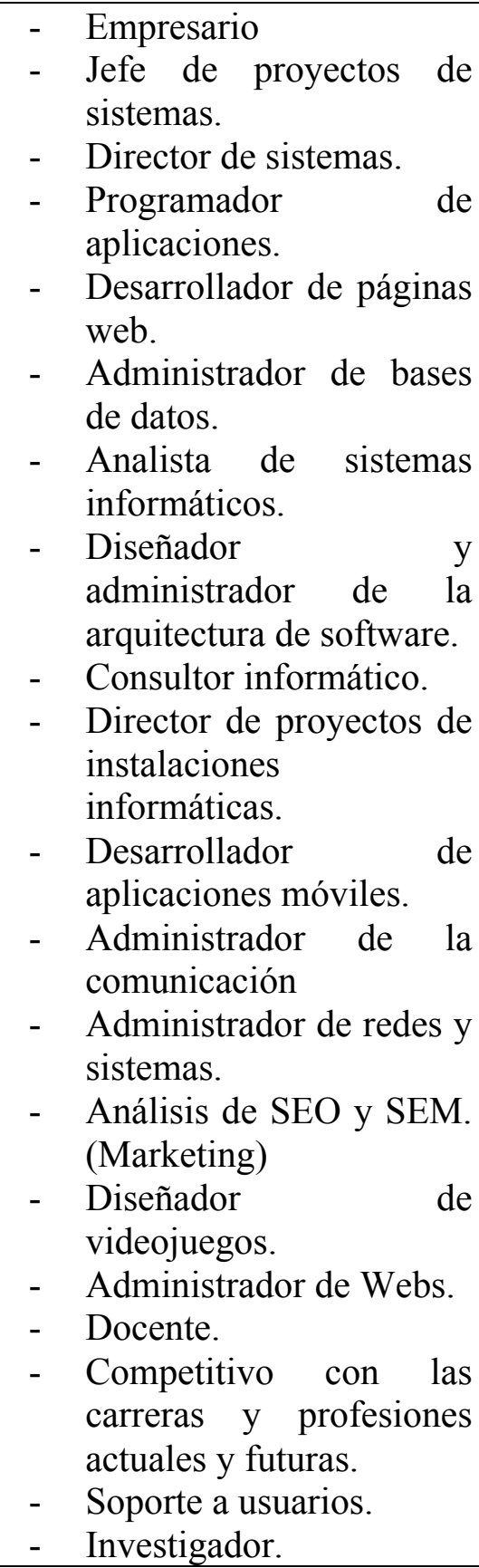 & $\begin{array}{l}\text { El Ingeniero de Sistemas } \\
\text { e Informática es } \\
\text { capacitado para } \\
\text { desempeñarse en: } \\
\text { - Gerencia y } \\
\text { asesoramiento en la } \\
\text { adecuación y desarrollo } \\
\text { de sistemas } \\
\text { informáticas. } \\
\text { - Desempeño en } \\
\text { instituciones educativas } \\
\text { tanto en el área docente } \\
\text { como administrativa. } \\
\text { - Auditoría de Sistemas } \\
\text { informáticos de toda } \\
\text { organización pública o } \\
\text { privada, industrial o de } \\
\text { servicio, que aplique } \\
\text { nuevos paradigmas de } \\
\text { administración y } \\
\text { sistemas. }\end{array}$ \\
\hline
\end{tabular}

Contacto: revistaeduca@umch.edu.pe ISSN : 2617-0337 revistas.umch.edu.pe/ 
- Planificador.

En el análisis correspondiente se presentan las funciones y campos laborales señalados por los empleadores que no fueron nombrados por los profesionales y docentes de esta área de estudio: Auditoria y Seguridad, Teoría de autómatas y teoría de diseño de algoritmos, Software y calidad, Conocimientos de inteligencia artificial o ingeniería del conocimiento, Instrumentación virtual, Robótica, Big Data y Arquitectura de microprocesadores. Mientras que los empleadores no plasmaron las funciones ni el campo laboral de la investigación y la docencia.

De acuerdo al segundo objetivo específico, construir el análisis funcional de la Carrera Profesional de Ingeniería de Sistemas e Informática de la universidad. A continuación se detalla el análisis los protocolos de textos de las entrevistas y la categorización y la subcategorización que emergió: Categoría Sol: Perfil Profesional del egresado de Ingeniería de Sistemas e Informática a partir de competencias. Y las Subcategorías: 1.- Ingeniería de Sistemas desde la visión del docente universitario y profesional (Objetivos - Funciones - Habilidades y destrezas - Campo laboral y Dimensiones). 2.- Ingeniería Informática desde la visión del docente universitario y profesional (Objetivos - Funciones - Habilidades y destrezas - Campo laboral y - Dimensiones). Se hizo por cada profesión por separado, ya que esa es la tendencia mundial.

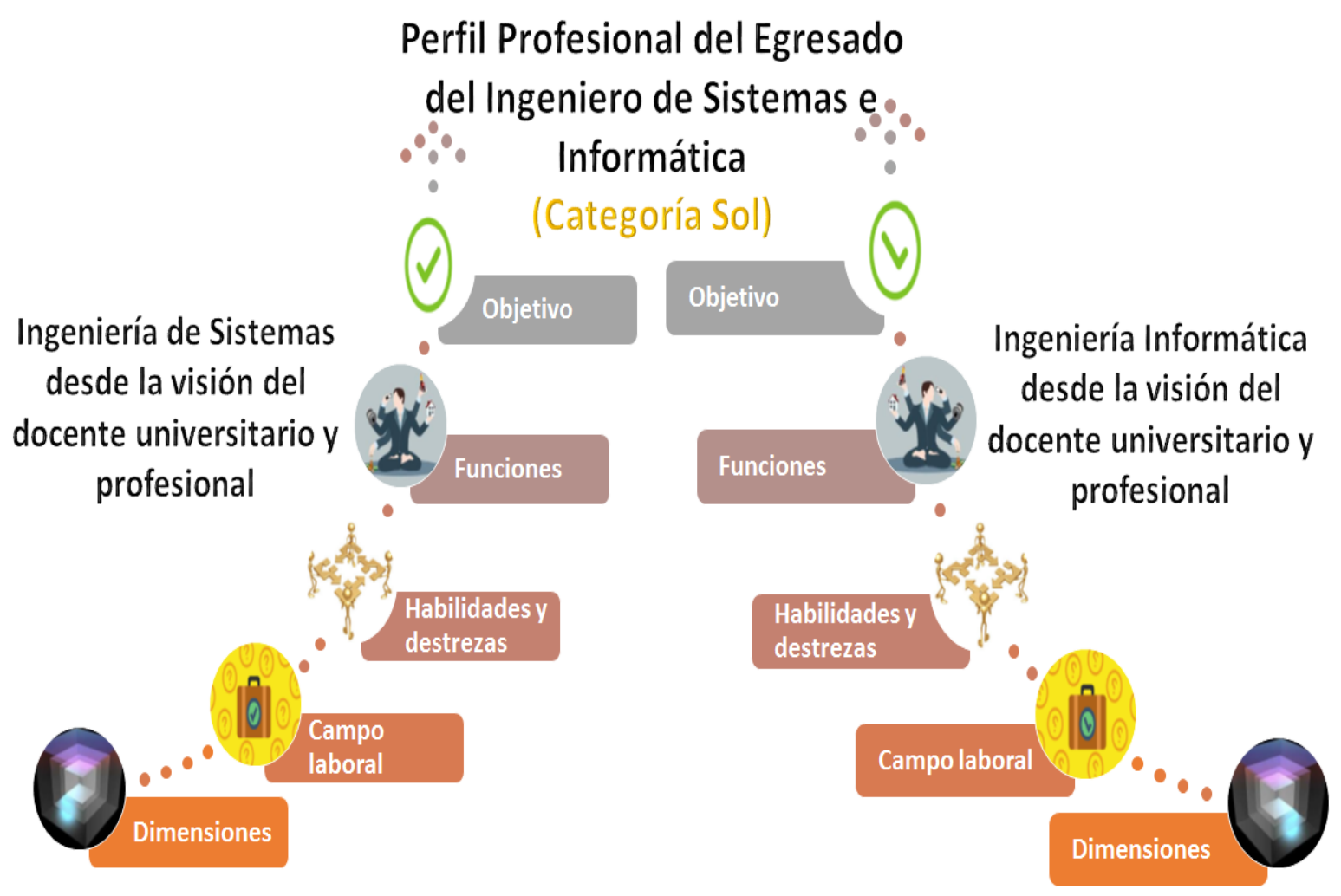

Contacto: revistaeduca@umch.edu.pe ISSN : 2617-0337 revistas.umch.edu.pe/ 
Figura 1. Perfil Profesional del Ingeniero de Sistemas e Informática - USDG, a partir de competencias.

Una vez culminado este análisis se procedió a implementar el método del análisis ocupacional sustentado por Santiváñez (2013). Resultados que se muestran a continuación y su respectiva explicación.

Con respecto al Ingeniero de Sistemas e Informática desde la visión los docentes, profesionales y empleadores se concluye lo siguiente:

1.- Definición: el Ingeniero de Sistemas e Informática, se ubica en el paradigma sistémico como una rama interdisciplinaria de la Ingeniería, que se apoya en las ciencias matemáticas, físicas, de los sistemas, de la computación, la electrónica, la ingeniería del software y las telecomunicaciones; con el propósito de comprender la realidad para el desarrollo de soluciones integrales de computo, de diseño, programación, comunicaciones y mantenimiento de sistemas, a través del procesamiento de la información de manera autómata, de la manipulación de los dispositivos informáticos, lo económico y la fuerza de la naturaleza de manera efectiva y compatible en beneficio de la humanidad.

\section{2.- Funciones:}

- Analizar las necesidades requeridas

- Diseñar, programar, aplicar y mantener (sistemas informáticos, páginas web, entre otros).

- Creador de aplicaciones móviles

- Administrar redes y sistemas de información.

- Creador de videojuegos y aplicaciones móviles.

- Encargado de plataformas de e-commerce.

- Experto en Telecomunicaciones.

- Gestor de proyectos y tecnologías.

- Analizar el rendimiento económico, la efectividad de los recursos humanos y el uso tecnológico vinculado a sus creaciones.

- Investigar para crear software y hardware en una empresa u organización.

3.- Objetivos: Administrar y gestionar los procesos gerenciales y técnicos y de solución tecnológica y teleinformática a través de estrategias innovadoras en la mejora de la productividad y competitividad.

\section{4.- Habilidades y destrezas:}

Contacto: revistaeduca@umch.edu.pe ISSN : 2617-0337 revistas.umch.edu.pe/ 
- Habilidad lógica

- Capacidad de análisis

- Desarrollo del pensamiento matemático y cálculos

- Capacidad de interpretativa

- Capacidad para ser Líder

- Trabajar en equipo

- Capacidad de adaptación al cambio tecnológico y empresarial

- Creativo

- Innovador

- Facilidad para comunicarse y establecer relaciones.

- Interés por planear, ejecutar o dirigir proyectos de investigación

- Trabajar bajo presión

- Ser ético, con valores morales

- Logro del éxito

- Resolutor de problemas

- Diseñar sistemas complejos

- Administrador de recursos tecnológicos y humanos.

- Seguridad Informática

- Calidad en Informática

- Tratamiento de datos

- Procesos de Inteligencia Artificial

- Desarrollador de Video Juegos

- Desarrollador de procesos de simulación

- Programación audiovisual

- Desarrollador de hardware

\section{5.- Campo laboral:}

- Empresario

- Jefe de proyectos de sistemas.

- Director de sistemas.

- Programador de aplicaciones.

- Desarrollador de páginas web.

- Administrador de bases de datos.

- Analista de sistemas informáticos.

- Diseñador y administrador de la arquitectura de software.

- Consultor informático.

- Director de proyectos de instalaciones informáticas.

- Desarrollador de aplicaciones móviles.

- Administrador de la comunicación

- Administrador de redes y sistemas.

- Análisis de SEO y SEM (Marketing).

- Diseñador de videojuegos.

- Administrador de Webs.

- Docente.

- Competitivo con las carreras y profesiones actuales y futuras.

- Soporte a usuarios.

Contacto: revistaeduca@umch.edu.pe ISSN : 2617-0337 revistas.umch.edu.pe/ 
- Investigador.

- Planificador

6.- Dimensiones: Ingeniería de Sistemas de Producto. Enterprise Systems Engineering. La Ingeniería de Sistemas de Servicio. Ciencia de la Computación y la Telemática. Con base a estos resultados se construye la Matriz ocupacional. Para el objetivo específico sobre comparar la funcionalidad de la Carrera Profesional de Ingeniería de Sistemas e Informática de la universidad con la demanda profesional de la Sociedad Peruana.

De acuerdo al tercer objetivo específico sobre comparar la funcionalidad de la Carrera Profesional de Ingeniería de Sistemas e Informática de la universidad con la demanda profesional de la Sociedad Peruana, se construye la matriz ocupacional.

\section{MATRIZ OCUPACIONAL}

\begin{tabular}{|r|}
\hline Campos ocupacionales \\
\hline Empresarial \\
\hline Gerencia \\
\hline Ingeniería del Software \\
\hline Ingeniería de Base de datos \\
\hline Gerencia de Proyectos Informáticos \\
\hline Ingeniería de Telecomunicaciones \\
\hline Videojuegos \\
\hline Auditoría de sistemas \\
\hline Seguridad Informática \\
\hline Inteligencia artificial, Big Data \\
\hline Robótica \\
\hline Ingeniería del Hardware \\
\hline TIC y Marketing \\
\hline
\end{tabular}
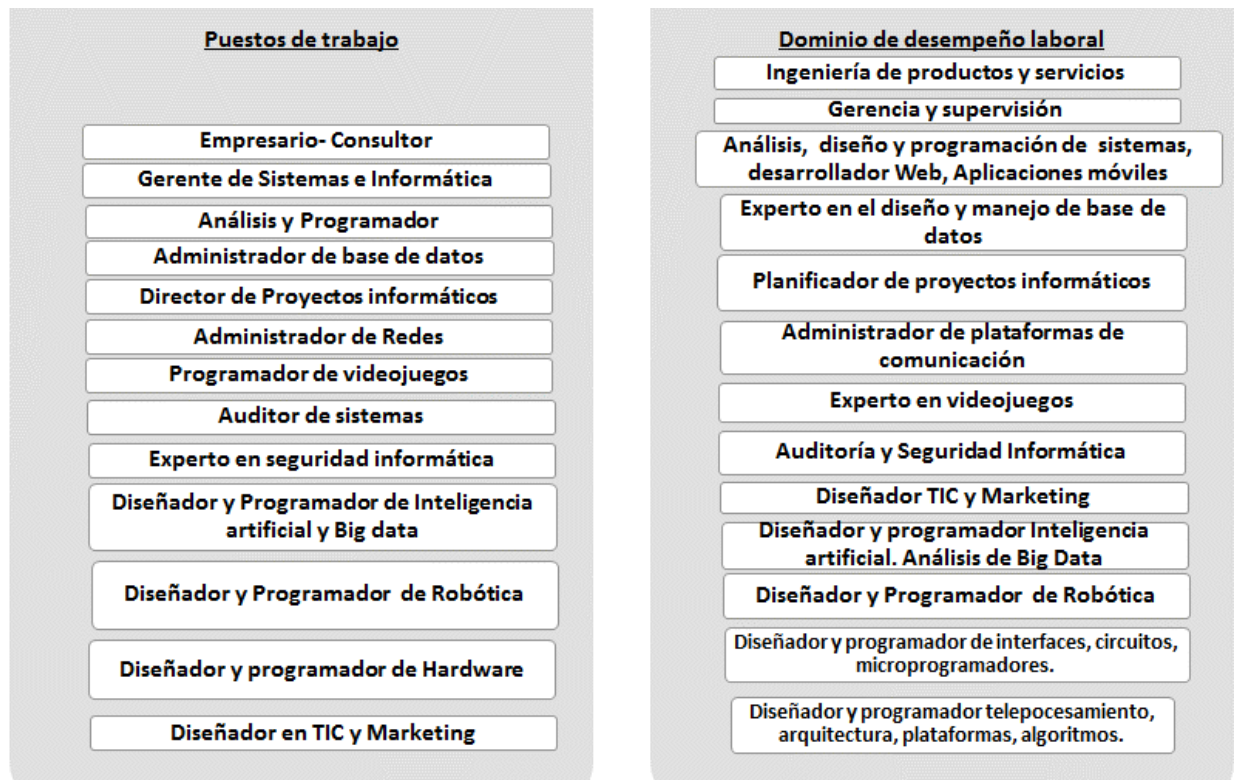

Figura 2. Matriz Ocupacional. Elaboración propia

De esta manera el perfil profesional del egresado de Ingeniería de Sistemas e Informática se conceptualiza, desde la visión de los profesionales y empleadores. Este perfil responde al objetivo general de la investigación que fue construir el perfil del egresado de la Carrera 
Profesional de Ingeniería de Sistemas e Informática de la Universidad Santo Domingo de Guzmán, tomando como principio la demanda profesional de la Sociedad Peruana.

El ingeniero en sistemas e informática es autodisciplinado y automatizado, con ética profesional, creativo e innovador y comunicador. Es un profesional líder con capacidad investigativa, alto nivel de abstracción lógica y con conocimientos integrales de las áreas de la ingeniería de software y hardware, la inteligencia artificial, las telecomunicaciones, las bases de datos y la seguridad informática. También actúa en calidad de gerente, director, analista y programador dentro del área de la informática y sistemas. Genera o participa en empresas para la ingeniería de productos, servicios, asesorías y auditorías. Está preparado para conformar grupos interdisciplinarios en la modelación, propuestas de soluciones informáticas complejas y proyectos escalables, interoperables, mantenibles y eficientes, aplicados en las tareas científicas, administrativas, técnicas y sociales.

A partir de estas premisas se podrá completar por ciclos tal como se muestra en la tabla 3, para la construcción de la malla curricular, lo cual forma parte de otra metodología.

\section{Tabla 3}

Dominio del desempeño laboral

Dominio de Desempeño Laboral

\section{Competencias \\ Desempeños \\ Específicas}

- Ingeniería de productos y servicios

- Gerencia, Dirección, Control y Supervisión

- Análisis, diseño y programación de sistemas, desarrollador Web, Aplicaciones móviles

- Experto en el diseño y manejo de base de datos

- Planificador de proyectos informáticos

- Administrador de plataformas operativas (Sistemas Operativos y de comunicación (Redes)

- Experto en videojuegos

- Auditoría

- Seguridad Informática

- Diseñador y programador Inteligencia artificial

- Analista de Big Data 
- Diseñador y Programador de Robótica

- Diseñador y programador de interfaces, circuitos, microprocesadores, microcontroladores y teleprocesamiento, arquitectura, plataformas y algoritmos.

\section{Conclusiones}

La investigación se concluyó con base en sus objetivos; con respecto al objetivo general sobre construir el perfil profesional del egresado de la Carrera Profesional de Ingeniería de Sistemas e Informática de la Universidad Santo Domingo de Guzmán, teniendo como principio la demanda profesional de la Sociedad Peruana, se tiene que:

El ingeniero en sistemas e informática es de carácter autodisciplinado y automatizado, con ética profesional, creativo e innovador y comunicador. Es un profesional líder con capacidad investigativa, alto nivel de abstracción lógica y con conocimientos integrales de las áreas de la ingeniería de software y hardware, la inteligencia artificial, las telecomunicaciones, las bases de datos y la seguridad informática. Para actuar con calidad de gerente, director, analista y programador dentro del área de la informática y sistemas. Genera o participa en empresas para la ingeniería de productos, servicios, asesorías y auditorías. Preparado para conformar grupos interdisciplinarios en la modelación, propuestas de soluciones informáticas complejas y proyectos escalables, interoperables, mantenibles y eficientes, aplicados en las tareas científicas, administrativas, técnicas y sociales.

De acuerdo con el primer objetivo específico, sobre caracterizar la demanda profesional del Ingeniero de Sistemas e Informática en la Sociedad Peruana, se presentaron las funciones planteadas por los empleadores, con respecto a las funciones del Ingeniero de Sistemas e Informática, a partir de la visión de los empleadores, se tiene: conocimientos de teoría de la información y telecomunicaciones (Redes, Auditoria y Seguridad), conocimientos de teoría de autómatas y teoría de diseño de algoritmos y lenguajes formales, conocimientos de ingeniería del software (Software, calidad, tiempo y coste), conocimientos de inteligencia artificial o ingeniería del conocimiento, competitiva industrial en el manejo de la información, conocimientos de Contacto: revistaeduca@umch.edu.pe ISSN : 2617-0337 revistas.umch.edu.pe/ 
electrónica para calcular y diseñar interfaces de comunicación y control entre computadores y diversos dispositivos mecánicos y eléctricos (Instrumentación virtual, Robótica), conocimientos de Organización industrial y empresarial, dirección y control TIC, conocimientos hardware para analizar y diseñar soluciones en el ámbito de la arquitectura de microprocesadores y analista de sistemas y manejador de Base de Datos, Marketing.

En concordancia con el segundo objetivo específico sobre construir el análisis funcional de la Carrera Profesional de Ingeniería de Sistemas e Informática de la universidad, se concluyó que el mismo sobre los campos ocupacionales, los puestos de trabajo y el dominio de desempeño laboral: Campos ocupacionales: Empresarial, Gerencia, Ingeniería del Software, Ingeniería de Base de datos, Gerencia de Proyectos Informáticos, Ingeniería de Telecomunicaciones, Videojuegos, Auditoría de sistemas, Seguridad Informática, Inteligencia artificial, Robótica e Ingeniería del Hardware, TIC y Marketing.

Puestos de trabajo: Empresario- Consultor, Gerente de Sistemas e Informática, Análisis y Programación, Administrador de base de datos, Director de Proyectos informáticos, Administrador de Redes, Programador de videojuegos, Auditor de sistemas, Experto en seguridad informática, Analista de Big Data, Diseñador y Programador de Inteligencia artificial, Diseñador y Programador de Robótica y Diseñador y programador de Hardware.

Dominio de desempeño laboral: Ingeniería de productos y servicios, gerencia y supervisión, Análisis, diseño y programación de sistemas, desarrollador Web, Aplicaciones móviles, experto en el diseño y manejo de base de datos, planificador de proyectos informáticos, administrador de plataformas de comunicación, experto en videojuegos, auditoría, seguridad informática, diseñador tic y marketing, diseñador y programador inteligencia artificial, Big Data, diseñador y programador de robótica y diseñador y programador de interfaces, circuitos, microprogramadores y teleprocesamiento, arquitectura, plataformas, algoritmos.

Por último, como tercer objetivo sobre comparar la funcionalidad de la Carrera Profesional de Ingeniería de Sistemas e Informática de la universidad con la demanda profesional de la sociedad peruana; se concluye que el Ingeniero de sistemas e Informática debe tener conocimientos en: teoría de la información y telecomunicaciones (Redes, Auditoria y Seguridad), teoría de autómatas y teoría de diseño de algoritmos y lenguajes formales, Ingeniería del software (Software, calidad, tiempo y coste), inteligencia artificial o ingeniería del conocimiento, competitiva industrial en el manejo de la información, electrónica para calcular y diseñar interfaces de comunicación y control

Contacto: revistaeduca@umch.edu.pe ISSN : 2617-0337 revistas.umch.edu.pe/ 
entre computadores y diversos dispositivos mecánicos y eléctricos (Instrumentación virtual, Robótica), organización industrial y empresarial, dirección y control TIC, hardware para analizar y diseñar soluciones en el ámbito de la arquitectura de microprocesadores; analista de sistemas y manejador de base de datos.

En el texto lo que señala aquellos conocimientos que si fueron tomados en cuenta por los empresarios como las funciones del Ingeniero de Sistemas e Informática, que debe desempeñar en el mundo laboral en la actualidad, más no fueron presentados por los informantes docentes, profesionales y estudiantes, tal como se muestra a continuación: Auditoria y seguridad, teoría de autómatas y teoría de diseño de algoritmos, software y calidad, Conocimientos de inteligencia artificial o ingeniería del conocimiento, Instrumentación virtual, robótica, Big Data y arquitectura de microprocesadores.

Actualmente las universidades están vinculadas a la formación por competencias, la formación integral y centrada en el estudiante, como un proceso educativo en el que deben garantizar una educación de calidad. El proceso de enseñanza y de aprendizaje debe estar permeado por los cambios tecnológicos y con una estructura global del proceso de producción de profesionales altamente competentes; por lo que el hombre debe permanecer en constante formación y actualización de los conocimientos a fin de desarrollar su creatividad, habilidades y destrezas para un desenvolvimiento en un mundo complejo.

Los profesionales de la Ingeniería de Sistemas e Informática se han convertido en solucionadores de problemas, en búsqueda de nuevas formas para hacer las cosas, por lo que esta profesión se compone de diversas disciplinas del saber. En este sentido la universidad debe reinventarse y estructurar procesos educativos dinámicos e interdisciplinarios, por lo cual desde el modelo educativo, el diseño curricular y los programas de estudio se debe innovar y estar a la vanguardia del mundo complejo.

\section{Referencias}

Anónimo (2012). Competencias y algo más. Disponible en: http://gestionescolar9.blogspot.com/2012_07_01_archive.html. Consultado: Junio, 2019.

Ballesteros, J. (2012). Competencias de los estudiantes de Ingeniería de Sistemas y Computación: Caso de estudio de Teoría General de Sistemas. En la Universidad Pedagógica y Tecnológica de Colombia. Tesis.

Contacto: revistaeduca@umch.edu.pe ISSN : 2617-0337 revistas.umch.edu.pe/ 
Braslavsky, C. (1999). Rehaciendo escuelas: hacia un nuevo paradigma en la educación latinoamericana. Buenos Aires: Santillana.

Bologna Declaration (1999). Bologna Declaration. Disponible en la página web del SIB: www.esib.org

Comisión Europea (1998). European Credit Transfer System ECTS Users' Guide. Publicado por la Comisión Europea (DG de Educación y Cultura) y disponible en: http:/europa.eu.int/comm/education/Sócrates/ects.html.

Comisión Europea (2001). ECTS Extensión «Questions and Answers». Diponible en la página web de la Comisión Europea: http:/europa.eu.int/comm/education/Sócrates/ectsfea.htm • Area. Este documento puede encontrarse en la página web del ESIB: www.esib.org

Delors. J. y otros (1996): La educación encierra un tesoro. Madrid: Santillana-Unesco.

Glasser, B. y Strauss, A. (1999). The Discovery of Grownder Theory. Strategies for cualitative. Reserach. Chicago: Aladine. Publishing Company.

Guardián, A. (1979). Modelo Metodológico de Diseño Cunicular. Costa Rica: Oficina de Publicaciones.

Guibert (2015). Tesis Las Competencias Profesionales y el Perfil Académico de los Estudiantes de la Facultad de Ciencias Contables, Económicas y Financieras. Perú: Universidad de San Martín De Porres.

Latorre, M. y Seco, C. (2016). Diseño Curricular Nuevo para una nueva sociedad-I Teoría. Lima: Santillana.

Maturana, H. (1997). Emociones y lenguaje en educación y política. México: Dolme.

Maturana, H. (1998). El conocer en investigación. Revista Iberoamericana de Educación. ISSSN 1985-5653. Universidad de Antioquia de México. México.

Molina (2000). Tesis: La competencia profesional en el ingeniero del nuevo milenio, como una investigación de Tesis Doctoral en el Instituto Superior Politécnico José Antonio Echeverría de la Habana, Facultad de Ingeniería Mecánica

ONU. (2016). Organización de las Naciones Unidas. Informe de los Objetivos de Desarollo Sostenible. Colombia: Unesco.

Pandit, R. (1995). La creación de la teoría. Una aplicación reciente del Método de Teoría Fundamentada. Informe cualitativo, Vol. 2, No. 4 Diciembre 1995 [documento en línea]. Disponible http://www.nova.edu/sss/ar/ar2-4/pandit.html. [Consultado: 2001, julio].

Pastor (2016). Investigación titulada Perfil del Ingeniero Industrial Actual en el Perú A Partir del Modelo Tuning Latinoamérica. Tesis de licenciatura en Ingeniería Industrial y de Sistemas) de la Universidad de Piura, Facultad de Ingeniería. Programa Académico de Ingeniería Industrial y de Sistemas.

Perrenoud, Ph. (2004). Diez nuevas competencias para enseñar. Barcelona: Graó

Contacto: revistaeduca@umch.edu.pe ISSN : 2617-0337 revistas.umch.edu.pe/ 
Reina (2008). Tesis doctoral: Competencias específicas de la carrera de Ingeniería Informática en la Universidad de Mendoza de la Universidad de Granada - Argentina.

Santivañez, V. (2013). Diseño Curricular A Partir De Competencias. Bogota: La U

Stenhouse L. (1984) Investigación y desarrollo del curriculum. Madrid: Morata.

Tejada Fernández, J. y Navío Gámez, A. (2005). El desarrollo y la gestión de competencias profesionales: una mirada desde la formación. Revista Iberoamericana 37 (02). Pp. 1-16. Recuperado de: http://www.rieoei.org/1089.htm

Tuning Project (2000). Página web: www.let.rug.nl/TuningProject o www.relint.deusto.ers/TuningProject/Molina, A. (2000). La Competencia Profesional en el Ingeniero del Nuevo Milenio. Revista Facultad de Ingeniería, núm. 8, julio-diciembre, 2000, pp. 65-71. Universidad de Tarapacá. Arica, Chile.

UNESCO (1998) Conferencia intergubernamental sobre políticas culturales para el desarrollo (Estocolmo, Suecia, 30 de marzo - 2 de abril de 1998). Informe final.

UNESCO (2007). Informe Mundial: Invertir en la diversidad cultural y el diálogo intercultural, enlace.

Zabalza, M.A. (2002). La enseñanza universitaria. El escenario y sus protagonistas. Madrid: Narcea.

Zabalza, M.A. (2003). Competencias docentes del profesorado universitario. Calidad y desarrollo profesional. Madrid: Narcea.

\section{Para citar este artículo:}

Sarmiento y J. Brito (2019). Perfil profesional del egresado de ingeniería de sistemas e informática por competencias. Revista Educa UMCH, 14(1). https://doi.org/10.15756/educaumch.v7i13.97 